\title{
Biocatalytic Asymmetric Hydrogen Transfer employing Rhodococcus ruber DSM 44541
}

\author{
Wolfgang Stampfer, Birgit Kosjek, Kurt Faber, Wolfgang Kroutil*
}

\section{$\underline{\text { Supporting Information }}$}

\section{Contents}

1. GC Analyses on achiral columns

2. GC Analyses on chiral columns

3. Optical rotation values

S5

4. Supporting References

S6

\section{GC Analyses on achiral columns}

Achiral columns were used for determination of activity.

The following achiral columns were used: column A: Hewlett Packard HP 1301 (30 m x $0.25 \mathrm{~mm} \times 0.25 \mu \mathrm{m}, 1.0$ bar $\mathrm{N}_{2}$ ); column B: Hewlett Packard HP 1701 (30 m x $0.25 \mathrm{~mm}$ x 0.25 $\mu \mathrm{m}, 1.0$ bar $\mathrm{N}_{2}$ ); column C: J\&W Scientific Agilent Technologies HP-1 (30 m x $0.25 \mathrm{~mm}$ x $0.25 \mu \mathrm{m}, 1.0$ bar $\left.\mathrm{N}_{2}\right)$; column D: Hewlett Packard HP-INNOwax (30 m x $0.25 \mathrm{~mm}$ x 0.25 $\mu \mathrm{m}, 1.0$ bar $\mathrm{N}_{2}$ ); column E: Chrompack Chirasil Dex (25 m x $0.32 \mathrm{~mm}$ x $0.25 \mu \mathrm{m}, 1.0$ bar $\left.\mathrm{H}_{2}\right)$; 
Table S1. Determination of conversion.

\begin{tabular}{|c|c|c|c|c|}
\hline \multirow[t]{2}{*}{ Compound } & \multirow[t]{2}{*}{ Program $^{a}$} & \multirow[t]{2}{*}{ Column } & \multicolumn{2}{|c|}{ Retention time [min] } \\
\hline & & & Ketone a & Alcohol b \\
\hline 1 & $100 / 9 / 30 / 240$ & A & 10.4 & 10.6 \\
\hline 2 & $90 / 9 / 30 / 240$ & A & 8.8 & 8.9 \\
\hline 3 & $80 / 5 / 10 / 105 / 0 / 30 / 240$ & A & 7.0 & 7.3 \\
\hline 4 & $80 / 5 / 10 / 105 / 0 / 30 / 240$ & $\mathrm{~A}$ & 4.8 & 5.1 \\
\hline 5 & $50 / 5 / 4 / 90 / 0 / 12 / 150$ & $\mathrm{E}$ & 7.4 & 11.7 \\
\hline 6 & $55 / 7 / 5 / 80 / 0 / 10 / 160$ & A & 6.8 & 7.1 \\
\hline 7 & $100 / 5 / 12 / 160 / 10$ & $\mathrm{E}$ & 2.1 & 5.5 \\
\hline 8 & $100 / 5 / 12 / 160 / 10$ & $\mathrm{E}$ & 7.8 & 8.8 \\
\hline 9 & 100 isotherm & A & 3.4 & 3.5 \\
\hline 10 & $100 / 12 / 5 / 160 / 10$ & $\mathrm{E}$ & 20.1 & 23.2 \\
\hline 11 & $90 / 12 / 15 / 130 / 0 / 30 / 250$ & $\mathrm{C}$ & 16.6 & 16.2 \\
\hline 12 & $120 / 10 / 20 / 160$ & $\mathrm{E}$ & 4.7 & 7.9 \\
\hline 13 & $70 / 2 / 5 / 120 / 2 / 160$ & $\mathrm{E}$ & 10.1 & 11.9 \\
\hline 14 & $120 / 0 / 20 / 250$ & $\mathrm{C}$ & 4.6 & 4.4 \\
\hline 15 & $100 / 9 / 30 / 240$ & $\mathrm{~A}$ & 12.1 & 12.2 \\
\hline 16 & 45 isotherm & A & 7.3 & 8.5 \\
\hline 17 & 45 isotherm & A & 4.0 & 4.5 \\
\hline 18 & 40 isotherm & A & 2.4 & 2.5 \\
\hline 19 & $80 / 5 / 10 / 105 / 0 / 30 / 240$ & $\mathrm{~A}$ & 7.8 & 8.4 \\
\hline 20 & $55 / 7 / 5 / 80 / 0 / 10 / 160$ & A & 6.7 & 6.8 \\
\hline 21 & $50 / 5 / 10 / 250$ & $\mathrm{D}$ & 10.1 & 12.1 \\
\hline 22 & $50 / 5 / 10 / 250$ & $\mathrm{D}$ & 8.0 & 10.2 \\
\hline 23 & $80 / 3 / 30 / 250$ & $\mathrm{~B}$ & 9.7 & 7.6 \\
\hline 24 & $80 / 3 / 30 / 250$ & $\mathrm{~B}$ & 9.5 & 8.8 \\
\hline 25 & $100 / 5 / 12 / 160 / 10$ & $\mathrm{E}$ & 3.8 & 6.0 \\
\hline 26 & $100 / 5 / 12 / 160 / 10$ & $\mathrm{E}$ & 5.5 & 8.8 \\
\hline
\end{tabular}




$\begin{array}{lllll}\mathbf{2 7} & 100 / 5 / 12 / 160 / 10 & \mathrm{E} & 3.5 & 9.9 \\ \mathbf{2 8} & 100 / 5 / 12 / 160 / 10 & \mathrm{E} & 3.6 & 6.7 \\ \mathbf{2 9} & 100 / 5 / 12 / 160 / 10 & \mathrm{E} & 3.2 & 7.3 \\ \mathbf{3 0} & 100 / 5 / 12 / 160 / 10 & \mathrm{E} & 2.7 & 8.7 \\ \mathbf{3 1} & 100 / 5 / 12 / 160 / 10 & \mathrm{E} & 3.4 & 8.8 \\ \mathbf{3 2} & 100 / 5 / 12 / 160 / 10 & \mathrm{E} & 2.9 & 8.6 \\ \mathbf{3 3} & 100 / 5 / 12 / 160 / 10 & \mathrm{E} & 8.0 & 9.0 \\ \mathbf{3 4} & 100 / 5 / 12 / 160 / 10 & \mathrm{E} & 6.1 & 8.7 \\ \mathbf{3 5} & 70 / 7.5 / 10 / 160 & \mathrm{E} & 10.2 & 12.9 \\ \mathbf{3 6} & 100 / 11 / 30 / 240 & \mathrm{~A} & 13.0 & 13.1 \\ \mathbf{3 7} & 80 / 17 / 12 / 160 & \mathrm{E} & 19.7 & 22.2 \\ \mathbf{3 8} & 80 / 17 / 12 / 160 & \mathrm{E} & 21.6 & 23.1 \\ \mathbf{3 9} & 70 / 7.5 / 10 / 160 & \mathrm{E} & 10.2 & 11.9 \\ \mathbf{4 0} & 100 / 13 / 10 / 160 & \mathrm{E} & 5.2 & 10.9\end{array}$

$a$ Program: initial temp. $\left[{ }^{\circ} \mathrm{C}\right] /$ time $[\mathrm{min}] /$ slope $\left[{ }^{\circ} \mathrm{C} / \mathrm{min}\right] /$ temp. $\left[{ }^{\circ} \mathrm{C}\right] /$ time $[\mathrm{min}] / \mathrm{slope}$ $\left[{ }^{\circ} \mathrm{C} / \mathrm{min}\right] /$ temp. $\left[{ }^{\circ} \mathrm{C}\right]$.

\section{GC Analyses on chiral columns}

Chiral columns used for determination of enantiomeric excess.

The following chiral columns were used: column E: Chrompack Chirasil Dex (25 m x 0.32 mm x $0.25 \mu \mathrm{m}, \mathrm{H}_{2}$ ); column F: Astec Chiraldex G-PN (30 m x $0.32 \mathrm{~mm}, 1.0$ bar $\mathrm{H}_{2}$ ).

The ee of $\mathbf{3 b}, \mathbf{5 b}, \mathbf{7 b}$ was determined as previously described. ${ }^{\mathrm{i}}$ 
Table S2. Determination of ee values

\begin{tabular}{|c|c|c|c|}
\hline Compound & Program $^{a}$ & Column & Retention time [min] \\
\hline $\mathbf{1 b}^{b}$ & $80 / 7 / 10 / 160$ & $\mathrm{~F}$ & $10.27(S), 10.38(R)$ \\
\hline $2 \mathbf{b}^{b}$ & $60 / 7 / 4 / 80 / 10 / 160$ & $\mathrm{~F}$ & $13.52(S), 13.69(R)$ \\
\hline $3 \mathbf{b}^{b}$ & $60 / 7 / 4 / 80 / 10 / 160$ & $\mathrm{~F}$ & $9.75(S), 10.18(R)$ \\
\hline $4 b^{b}$ & $60 / 7 / 4 / 80 / 10 / 160$ & $\mathrm{~F}$ & $8.48(S), 9.08(R)$ \\
\hline $5 \mathbf{b}$ & $50 / 5 / 4 / 90 / 12 / 150$ & $\mathrm{E}$ & $11.72(S), 12.24(R)$ \\
\hline $\mathbf{6 b}^{b}$ & $55 / 7 / 5 / 80 / 10 / 160$ & $\mathrm{~F}$ & $10.35(S), 10.58(R)$ \\
\hline $7 b$ & $100 / 5 / 12 / 160 / 10$ & $\mathrm{E}$ & $5.53(R), 5.99(S)$ \\
\hline $8 b$ & $100 / 5 / 12 / 160 / 10$ & $\mathrm{E}$ & $8.85(R), 9.01(S)$ \\
\hline $9 \mathbf{b}^{b}$ & 90 isotherm & $\mathrm{E}$ & $5.93(S), 8.19(R)$ \\
\hline $10 b$ & $100 / 12 / 5 / 160 / 10$ & $\mathrm{E}$ & $23.18(R), 23.56(S)$ \\
\hline $11 b$ & $100 / 12 / 5 / 160 / 10$ & $\mathrm{E}$ & $9.75(S), 9.99(R)$ \\
\hline $12 b$ & $120 / 10 / 20 / 160$ & $\mathrm{E}$ & $7.85(S), 8.38(R)$ \\
\hline $13 b$ & $70 / 2 / 5 / 120 / 0 / 12 / 160$ & $\mathrm{E}$ & $11.9,12.1$ \\
\hline $14 b^{b}$ & $80 / 6.5 / 10 / 160 / 10$ & $\mathrm{E}$ & $13.31(S), 13.47(R)$ \\
\hline $15 b^{b}$ & $55 / 0 / 10 / 140 / 1.5$ & $\mathrm{E}$ & $8.95,9.18$ \\
\hline $16 \mathbf{b}^{b}$ & 40 isotherm & $\mathrm{F}$ & $7.29(S), 8.53(R)$ \\
\hline $17 b^{b}$ & 40 isotherm & $\mathrm{F}$ & $3.00(S), 3.24(R)$ \\
\hline $18 b^{b}$ & 40 isotherm & $\mathrm{F}$ & $2.07(S), 2.34(R)$ \\
\hline $24 b$ & $115 / 15 / 12 / 160 / 0$ & $\mathrm{E}$ & $26.63(R), 26.97(S)$ \\
\hline $25 b$ & $100 / 5 / 12 / 160 / 10$ & $\mathrm{E}$ & $6.00(R), 6.57(S)$ \\
\hline $26 b$ & $100 / 5 / 12 / 160 / 10$ & $\mathrm{E}$ & $8.76(R), 9.06(S)$ \\
\hline $27 b$ & $100 / 5 / 12 / 160 / 10$ & $\mathrm{E}$ & $9.88(R), 10.10(S)$ \\
\hline $28 b$ & $100 / 5 / 12 / 160 / 10$ & $\mathrm{E}$ & $6.72(R), 7.16(S)$ \\
\hline $29 b$ & $100 / 5 / 12 / 160 / 10$ & $\mathrm{E}$ & $7.28(R), 7.49(S)$ \\
\hline $31 b$ & $100 / 5 / 12 / 160 / 10$ & $\mathrm{E}$ & $8.76(R), 8.99(S)$ \\
\hline $33 b$ & $100 / 5 / 12 / 160 / 10$ & $\mathrm{E}$ & $9.03(R), 9.18(S)$ \\
\hline $35 b$ & 70/7.5/10/160 & $\mathrm{E}$ & $12.88(R), 13.03(S)$ \\
\hline
\end{tabular}




$\begin{array}{cccc}\text { 37b } & 80 / 17 / 12 / 160 & \text { E } & 22.20(S), 22.29(R) \\ \text { 39b } & 70 / 7.5 / 10 / 160 & \text { E } & 11.88(S), 11.99(R) \\ \text { 40b } & 100 / 13 / 10 / 160 & \text { E } & 10.89(S), 11.34(R)\end{array}$

$a$ Program: initial temp. $\left[{ }^{\circ} \mathrm{C}\right] /$ time $[\mathrm{min}] / \mathrm{slope}\left[{ }^{\circ} \mathrm{C} / \mathrm{min}\right] /$ temp. $\left[{ }^{\circ} \mathrm{C}\right] /$ time $[\mathrm{min}] / \mathrm{slope}$ $\left[{ }^{\circ} \mathrm{C} / \mathrm{min}\right] /$ temp. $\left[{ }^{\circ} \mathrm{C}\right]$.

$b$ Enantiomeric excess determined as the corresponding acetate derivative.

\section{Optical rotation values}

(S)-2-Decanol 1b: $[\alpha]_{\mathrm{D}}{ }^{20}+6.97$ (c 1.00, EtOH) 58.3\% ee, lit. ${ }^{\mathrm{ii}}+10.3$ (c 0.80, EtOH) $99 \%$ ee.

$(S)-$ Sulcatol 5b: $[\alpha]_{\mathrm{D}}{ }^{20}+9.55\left(\right.$ c $\left.2.4, \mathrm{CHCl}_{3}\right) 99 \%$ ee, lit. ${ }^{\mathrm{ii}}+12.0\left(\right.$ c $\left.2.93, \mathrm{CHCl}_{3}\right) 99 \%$ ee.

(S)-3-Octanol 6b: $[\alpha]_{\mathrm{D}}{ }^{22} 9.15\left(\mathrm{c} 4.33, \mathrm{CHCl}_{3}\right) 97 \%$ ee, lit. ${ }^{\mathrm{iii}} 8.8\left(\mathrm{c} 0.49 \mathrm{CHCl}_{3}\right)$.

(R)-1-Cyclohexyl ethanol 9b: $[\alpha]_{\mathrm{D}}{ }^{20} 8.87$ (c 1.01, $\left.\mathrm{Et}_{2} \mathrm{O}\right) 99 \%$ ee, lit. ${ }^{\text {iv }}-7.79$ (c 3.10, $\mathrm{Et}_{2} \mathrm{O}$ ) $96 \%$ ee.

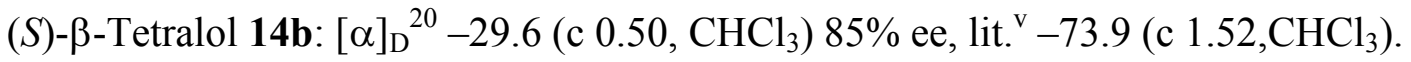

(S)-1-(4-Hydroxyphenyl) ethanol 24b: $[\alpha]_{\mathrm{D}}{ }^{20}-29.61$ (c 0.89, EtOH), 98\% ee, lit. ${ }^{\mathrm{vi}}-47.5$ (c 4.98, EtOH).

(S)-1-(4-Fluorophenyl) ethanol 25b: $[\alpha]_{\mathrm{D}}{ }^{20}-33.1$ (c 1.00, MeOH abs), 98\% ee, lit. ${ }^{\text {ii }}-37.7$ (c $0.931, \mathrm{MeOH}) 99 \%$ ee.

(R)-1-(4-Chlorophenyl) ethanol 26b: $[\alpha]_{\mathrm{D}}{ }^{20} 36.73$ (c 1.00, $\mathrm{CHCl}_{3}$ ), 88\% ee, lit. ${ }^{\text {vii }}-48.8$ (c 3.13, $\left.\mathrm{CHCl}_{3}\right)$ for $(S)$-enantiomer.

(R)-1-(4-Bromophenyl) ethanol 27b: $[\alpha]_{\mathrm{D}}{ }^{20} 27.64\left(\mathrm{c} 1.00, \mathrm{CHCl}_{3}\right), 89.4 \%$ ee, lit. ${ }^{\text {ii }}-37.9$ (c $\left.1.13, \mathrm{CHCl}_{3}\right) 99 \%$ ee $(S)$.

(R)-1-(4-Methylphenyl) ethanol 28b: $[\alpha]_{\mathrm{D}}{ }^{20} 27.54$ (c 1.00, MeOH), 87\% ee, lit. ${ }^{\mathrm{ii}}-43.5$ (c 0.994, $\mathrm{MeOH}) 99 \%$ ee $(S)$. 
(R)-1-(3-Methylphenyl) ethanol 29b: $[\alpha]_{\mathrm{D}}{ }^{20} 30.59$ (c 1.00, EtOH), 96\% ee, lit. ${ }^{\mathrm{ii}}-39.8$ (c 0.944, EtOH) $99 \%$ ee $(S)$.

(R)-1-(3-Chlorophenyl) ethanol 31b: $[\alpha]_{\mathrm{D}}{ }^{20} 27.73$ (c 3.5, $\mathrm{CHCl}_{3}$ ), 87\% ee, lit. ${ }^{\mathrm{ii}}-43.5$ (c $\left.1.08, \mathrm{CHCl}_{3}\right) 99 \%$ ee $(S)$.

(S)-1-(3-Methoxyphenyl) ethanol 33b: $[\alpha]_{\mathrm{D}}{ }^{20}-28.80$ (c 3.00, MeOH), 98\% ee, lit. ${ }^{\text {ii }}-34.9$ (c $0.85, \mathrm{MeOH}$ abs) $99 \%$ ee $(S)$.

(R)-4-Phenyl-2-butanol 40b: $[\alpha]_{\mathrm{D}}{ }^{20}-15.2\left(\mathrm{c} 1.21 \mathrm{CHCl}_{3}\right.$ ), 94\% ee, lit. ${ }^{\text {viii }} 17.4$ (c 1.80 , $\left.\mathrm{CHCl}_{3}\right)$ for the $(S)$-enantiomer.

\section{Supporting References}

i Stampfer, W.; Kosjek, B.; Moitzi, C.; Kroutil, W.; Faber, K. Angew. Chem. Int. Ed. Engl. 2002, 41, 1014-1017.

ii Nakamura, K.; Matsuda, T. J. Org. Chem. 1998, 63, 8957-8964.

iii Itoh, T.; Mitsukura, K.; Kanphai, W.; Takagi, Y.; Kihara, H.; Tsukube, H. J. Org. Chem. 1997, 62, 91656172.

iv Uozumi, Y.; Hayashi, T. J. Am. Chem. Soc. 1991, 113, 9887-9888.

v Arakawa, H.; Torimoto, N.; Masui, Y. Tetrahedron Lett. 1968, 4115-4117.

vi Everhart E. T.; Craig J. C. J. Chem Soc. Perkin Trans 1 1991, 1701-1707.

vii Akakabe, Y.; Takahashi, M.; Kamezawa, M.; Kikuchi, K.; Tachibana, H. J. Chem. Soc. Perkin Trans. 1 1995, 1295-1298.

viii Nakamura, K.; Inoue, Y.; Matsuda, T.; Misawa, I. J. Chem. Soc. Perkin Trans 1 1999, 2397-2402. 